\title{
UHRF1 expression is upregulated and associated with cellular proliferation in colorectal cancer
}

\author{
YASUHIDE KOFUNATO $^{1}$, KENSUKE KUMAMOTO ${ }^{1}$, KATSUHARU SAITOU $^{1}$, SUGURU HAYASE $^{1}$, \\ HIROKAZU OKAYAMA ${ }^{1}$, KOTARO MIYAMOTO ${ }^{1}$, YU SATO ${ }^{1}$, KYOKO KATAKURA $^{2}$, IZUMI NAKAMURA ${ }^{1}$, \\ SHINJI OHKI ${ }^{1}$, YOSHIHISA KOYAMA ${ }^{1}$, MOTOKO UNOKI ${ }^{3}$ and SEIICHI TAKENOSHITA ${ }^{1}$ \\ Departments of ${ }^{1}$ Organ Regulatory Surgery, ${ }^{2}$ Gastroenterology and Rheumatology, Fukushima \\ Medical University School of Medicine, Fukushima 960-1295; ${ }^{3}$ Division of Epigenomics, \\ Medical Institute of Bioregulation, Kyushu University, Fukuoka 812-8582, Japan
}

Received May 22, 2012; Accepted July 27, 2012

DOI: $10.3892 / o r .2012 .2064$

\begin{abstract}
Ubiquitin-like with PHD and ring-finger domain 1 (UHRF1) binds to methylated promoters of a number of tumor-suppressor genes, including $\mathrm{p} 16^{\mathrm{INK} 4 \mathrm{~A}}$ and $\mathrm{p} 14^{\mathrm{ARF}}$, by forming complexes with DNA methyltransferases and HDAC1, resulting in the induction of carcinogenesis. Altered UHRF1 expression has been demonstrated in various types of cancers. Previous reports indicate that UHRF1 expression is regulated by E2F-1 expression. We investigated UHRF1 expression using immunohistochemical staining in 231 colorectal cancer and 40 adenoma specimens, analyzed the relationship between UHRF1 expression and clinicopathological findings and the association between UHRF1 and E2F-1 expression. To better understand the biological function of UHRF1 in colorectal cancer, knockdown of UHRF1 expression was performed using siRNA methods. High UHRF1 expression was observed in 152 of 231 $(65.8 \%)$ colorectal cancer patients, and was detected in 35 of 40 adenoma specimens samples $(87.5 \%)$. UHRF1 staining was detected in the nucleus of cancer cells, while it was not detected in colonic normal mucosa. High UHRF1 expression was significantly observed in right compared with left hemicolon cancer $(\mathrm{P}=0.008)$. Moreover, high UHRF1 expression tended to be associated with depth of invasion $(\mathrm{P}=0.051)$. UHRF1 expression was significantly associated with E2F-1 expression $(\mathrm{P}<0.0001)$. Knockdown of UHRF1 expression suppressed cellular growth in colon cancer cell lines, HCT116 and SW620. In conclusion, we demonstrated that UHRF1 expression was upregulated in approximately two-thirds of colorectal cancer specimens and was particularly expressed in right compared
\end{abstract}

Correspondence to: Dr Kensuke Kumamoto, Department of Organ Regulatory Surgery, Fukushima Medical University School of Medicine, 1 Hikarigaoka, Fukushima 960-1295, Japan

E-mail:kumamotk@fmu.ac.jp

Key words: ubiquitin-like with PHD and ring-finger domain 1, colorectal cancer, E2F-1 with left hemicolon cancer. Moreover, knockdown of UHRF1 expression induced growth inhibition in colon cancer cell lines. UHRF1 may be involved in cellular proliferation and molecular pathogenesis of colorectal cancer in the right hemicolon.

\section{Introduction}

Recently, molecular-targeted drugs including bevacizumab (1), cetuximab (2) and panitumumab (3) have attracted increased attention for improvement of survival outcome following conventional chemotherapy in refractory colorectal cancer. A number of patients receiving chemotherapy gain the additional benefit of conversion therapy when the chemotherapeutic effect is observed remarkably in refractory colorectal cancer patients. However, $40-50 \%$ of patients who receive chemotherapy may experience adverse events. Regarding anti-EGFR antibodies, the efficacy is limited to colorectal patients with K-ras wild-type (4). Therefore, the development of predictive markers for efficacy of chemotherapy and novel molecular targets are required for colorectal cancer treatment.

It has been reported that ubiquitin-like with PHD and ring-finger domain 1 (UHRF1) expression, also known as ICBP90 and Np95, is upregulated in various types of cancers, including breast, lung, pancreatic, astrocytomas, cervical and bladder cancer (5-12). These common alterations in cancer cells imply the biological functions of UHRF1 that are associated with rapid cellular progression and DNA repair processes (13). In fact, decreasing UHRF1 expression suppresses cellular proliferation by inhibiting G1-S transition $(7,14,15)$. Thus, UHRF1 is considered to play an essential role in cellular proliferation.

UHRF1 plays a pivotal role in carcinogenesis via gene silencing mechanism. UHRF1 possesses several domains, which are associated with DNA methylation and histone methylation by recognizing hemi-methylated DNA and recruiting DNA methyltransferase 1 (DNMT1) (16-20). UHRF1 co-operates with histone deacetylase 1 (HDAC1) and methyltransferase G9a, which methylates histone $\mathrm{H} 3 \mathrm{~K}$ 9, and induces heterochromatin formation through these interactions (21). In cancer cells, UHRF1 localizes on methylated promoters of a 
Table I. Clinicopathological findings of 231 patients with colon cancer.

\begin{tabular}{|c|c|}
\hline Characteristics & $\mathrm{n}$ \\
\hline \multicolumn{2}{|l|}{ Gender } \\
\hline Male & 136 \\
\hline Female & 95 \\
\hline Median age (years) & $66(24-89$ \\
\hline \multicolumn{2}{|l|}{ Tumor location } \\
\hline Right hemicolon & 79 \\
\hline Left hemicolon & 152 \\
\hline \multicolumn{2}{|l|}{ Tumor differentiation } \\
\hline Differentiated & 201 \\
\hline Undifferentiated & 30 \\
\hline \multicolumn{2}{|l|}{ Depth of invasion } \\
\hline $\mathrm{T} 1$ & 8 \\
\hline $\mathrm{T} 2$ & 45 \\
\hline $\mathrm{T} 3$ & 162 \\
\hline $\mathrm{T} 4$ & 16 \\
\hline \multicolumn{2}{|c|}{ Lymph node metastasis } \\
\hline $\mathrm{NX}$ & 7 \\
\hline N0 & 138 \\
\hline N1 & 58 \\
\hline $\mathrm{N} 2$ & 21 \\
\hline N3 & 7 \\
\hline \multicolumn{2}{|l|}{ UICC stage } \\
\hline 0 & 5 \\
\hline $\mathrm{I}$ & 36 \\
\hline II & 85 \\
\hline III & 67 \\
\hline IV & 38 \\
\hline
\end{tabular}

number of tumor-suppressor genes, including $\mathrm{p} 16^{\mathrm{INK} 4 \mathrm{~A}}, \mathrm{p} 14^{\mathrm{ARF}}$ and BRCA, through complexes with HDAC1 and DNMT1 $(7,21,22)$. A previous report showed that UHRF1 is involved in de novo DNA methyltransferases, DNMT3a and DNMT3b (23). Therefore, UHRF1 may recruit these de novo DNA methyltransferases to methylated tumor-suppressor promoters further suppressing the expression of tumor-suppressor genes, resulting in the contribution to carcinogenesis.

Regarding the regulation of UHRF1 expression, E2F-1 induces the expression of UHRF1 through binding to the UHRF1 promoter $(6,7,24)$. Moreover, the tumor-suppressor p53 has been revealed to suppress UHRF1 expression through the inactivation of E2F-1 (14). Thus, the overexpression of UHRF1 in various types of cancers is considered to be associated with the p53-E2F-1 pathway.

Although enhanced UHRF1 expression has been demonstrated in various types of cancers, there is no evidence to demonstrate the relationship between UHRF1 expression and gastrointestinal cancer. In the present study, UHRF1 expression was evaluated using an immunohistochemical method in 231 colorectal cancer cases and 40 colonic adenomas. The relationship between UHRF1 expression and clinicopathological factors was analyzed. We further examined the correlation between UHRF1 and E2F-1 expression.

\section{Materials and methods}

Tissue samples. A total of 231 surgical specimens of colorectal cancer, obtained from the Fukushima Medical University Hospital from January 1990 to March 2007, were used for immunohistochemical experiments. The clinical characteristics are shown in Table I. The carcinomas at the time of the primary tumor resection were staged according to the UICC classification. An additional 40 adenoma samples were obtained from the Fukushima Medical University Hospital from January 2007 to March 2008. This study was performed in accordance with the Ethical Guidelines for Clinical Research with the approval of the Institutional Ethics Committee. Informed consent was obtained from the individuals included in the study.

Immunohistochemical staining and evaluation of UHRF1 and E2F-1 expression. Immunohistochemical staining of UHRF1 and E2F-1 was performed as previously described (12). Briefly, paraffin sections were incubated at $4^{\circ} \mathrm{C}$ overnight with mouse monoclonal anti-human UHRF1 antibody (BD Transduction Laboratories, San Jose, CA, USA) and mouse monoclonal anti-E2F-1 antibody (Santa Cruz Biotechnology, Santa Cruz, $\mathrm{CA})$ at room temperature for $1 \mathrm{~h}$. These proteins were visualized by EnVision System-HRP (Dako). Immunohistochemical evaluations were performed by two investigators (Y.K. and K.K.) independently without prior information of the clinicopathological features. For the microscopic analysis, at least 200 cancer cells were examined to determine whether the cells were positive for UHRF1 and E2F-1 at high power (x200) after screening for the areas with the highest intensity of staining at lower power (x40). A high expression was defined when positive staining cancer cells were observed in $>20 \%$ of at least 200 cancer cells, and a low expression was defined when $<20 \%$ of at least 200 cancer cells were stained.

Cell culture. Twelve human colon cancer cell lines, Colo 201, Colo 205, HCT15, HCT116, LS174T, LS180, LoVo, RKO, SW48, SW480, SW620 and SW837, were originally obtained from the American Type Culture Collection (Rockville, MD, USA). These cells were grown at $37^{\circ} \mathrm{C}$ under the presence of $5 \% \mathrm{CO}_{2}$ in the recommended culture media.

Western blotting. Protein lysates were prepared in ice cold lysis buffer (50 mM Tris $\mathrm{HCl}, \mathrm{pH} 7.5,150 \mathrm{mM} \mathrm{NaCl}, 1 \%$ Triton X-100, $50 \mathrm{mM} \mathrm{NaF}, 10 \mathrm{mM}$ sodium pyrophosphate, $25 \mathrm{mM} \beta$-glycerophosphate, protease and phosphatase inhibitors). Protein aliquots were separated by Tris-Glycine gels, and transferred onto nitrocellulose membranes. The protein blots were incubated with a primary antibody for $1 \mathrm{~h}$ at room temperature, and then incubated with a horseradish peroxidase-conjugated secondary antibody (Santa Cruz Biotechnology) for $30 \mathrm{~min}$ at room temperature. Bound antibodies were detected by enhanced chemiluminescence (ECL) detection reagents (Thermo Fisher Scientific Inc., Waltham, MA, USA) and visualized by autoradiography. The 

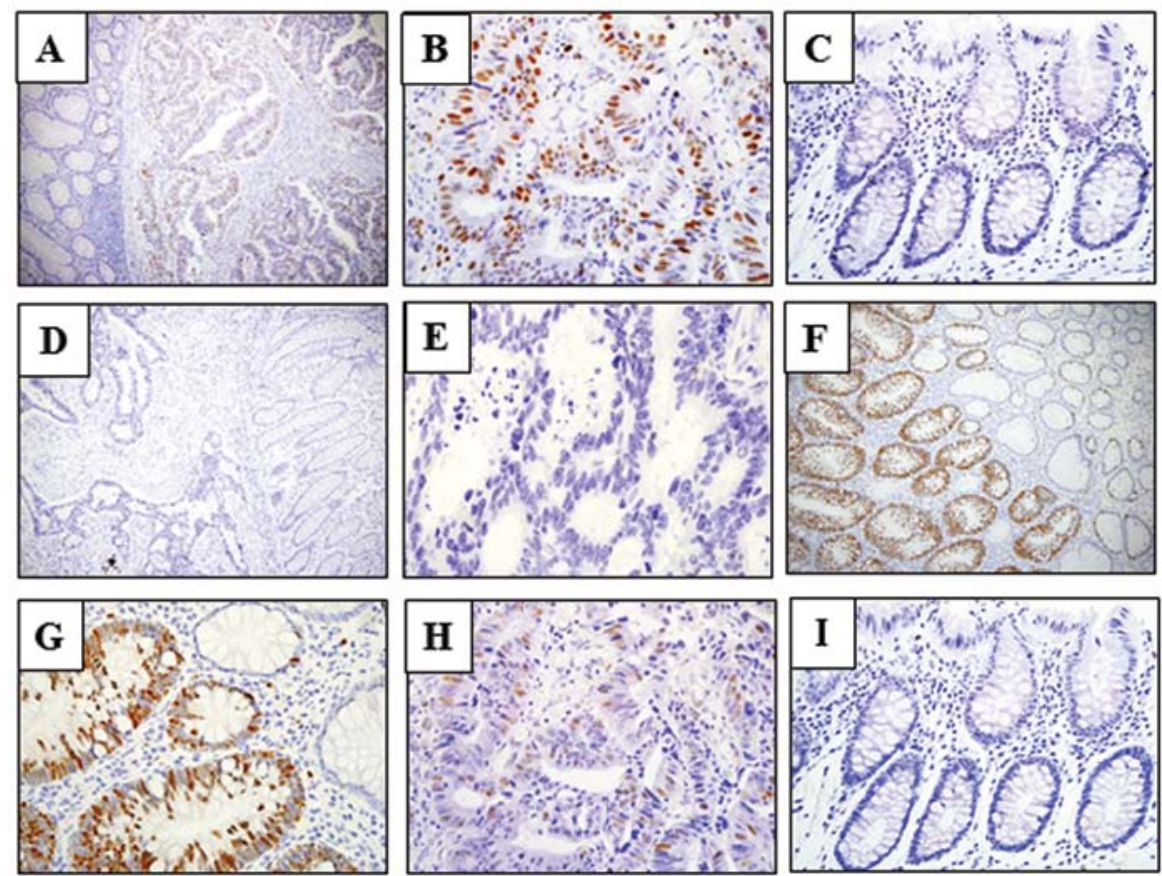

Figure 1. Expression of UHRF1 and E2F-1 in colonic adenoma and colorectal cancer tissues. Typical staining of UHRF1 expression in colorectal cancer (A-E) and colonic adenoma ( $\mathrm{F}$ and $\mathrm{G}$ ) and E2F-1 expression ( $\mathrm{H}$ and $\mathrm{I}$ ) in colorectal cancer. A and D are conjugated images with tumor and normal mucosa (magnification, x40). (B) High UHRF1 expression in cancer tissues (magnification, x200); (C) normal mucosa (magnification, x200); (E) low UHRF1 expression in cancer tissues (magnification,x200); ( $\mathrm{F}$ and G) high UHRF1 expression in colonic adenoma with normal mucosa (magnification, $\mathrm{x} 40$, x200, respectively); (H) high E2F-1 expression in cancer tissues (magnification, x200); (I) normal mucosa (magnification, x200).

primary antibodies used for the western blot analysis were mouse monoclonal anti-UHRF1 antibody (BD Transduction Laboratories) and mouse monoclonal anti- $\beta$-actin antibody (Santa Cruz Biotechnology).

Real-time reverse transcription-PCR (RT-PCR) analysis of mRNA expression. Total RNA from cells was harvested using TRIzol (Invitrogen, Carlsbad, CA, USA) according to the manufacturer's instructions. Total RNA $(5 \mu \mathrm{g})$ was used for the synthesis of first-strand cDNA using SuperScript III First-strand cDNA Synthesis kit (Invitrogen) following the manufacturer's instructions. RT-PCR analysis was performed using ABI PRISM 7500 (Applied Biosystems, Foster City, CA, USA) with TaqMan probes provided by the manufacturer. TaqMan probes of UHRF1 (Hs00273589_m1) and $\beta$-actin (Hs99999903_m1) were used for RT-PCR analysis. The relative amount of UHRF1 transcripts was normalized by the amount of $\beta$-actin transcripts used as the internal control in the same sample, and described as the ratio of UHRF1/ $\beta$-actin.

siRNA experiments. The siRNA oligos (Invitrogen) were designed as follows: UHRF1 siRNA, 5'-GCGCAATGTCAA GGGTGGCAAGAAT-3'; scrambled siRNA, 5'-GCGTATG ACGAGGGTCGAAGACAAT-3'. Each siRNA oligos was transfected into colon cancer cells using the RNAiMAX reagent (Invitrogen) according to the manufacturer's instructions. Briefly, cells were resuspended to an appropriate concentration $\left(1 \times 10^{5}\right.$ cells $\left./ \mathrm{ml}\right)$. The cell suspensions $(2 \mathrm{ml})$ were dispensed on a 6-well culture plate. Cells were the treated with $40 \mathrm{nM}$ UHRF1 siRNA or $40 \mathrm{nM}$ scrambled siRNA, and harvested at $48 \mathrm{~h}$ after treatment of siRNAs.
Cell counting. Cell counting was performed using a Cell Counting kit-8 (Dojindo, Kumamoto, Japan). Approximately 5,000 cells, which were treated with $40 \mathrm{nM}$ UHRF1 siRNA or $40 \mathrm{nM}$ scrambled siRNA, were dispensed onto a well of 96-well plate. After $48 \mathrm{~h}$ of incubation, the absorbance $(450 \mathrm{~nm})$ was measured using a microplate spectrophotometer (Bio-Rad Laboratories; Benchmark Plus).

Statistical analysis. The statistical analysis of the relationship between UHRF1 expression and clinicopathological findings was performed using Chi-square test, t-test, or Mann-Whitney test. The Chi-square test was performed to evaluate the relationship between UHRF1 and E2F-1 expression. The cell viability tests were performed in triplicate. Results are presented as the means \pm standard deviation. $\mathrm{P}<0.05$ were considered to indicate a statistically significant difference. Statistical analysis was performed with Prism 5 software (GraphPad Software, Inc., La Jolla, CA, USA).

\section{Results}

UHRF1 expression was upregulated in colon cancer. Of 231 colon cancer cases, high UHRF1 expression was detected in $152(65.8 \%)$ by immunohistochemical evaluation. UHRF1 expression was detected in cancer cells, while no expression was observed in normal mucosa (Fig. 1A-E). The cellular localization of UHRF1 was nuclear. We further examined UHRF1 expression in 40 colonic adenomas. UHRF1 expression was positively detected in 35 out of 40 samples (87.5\%) (Fig. 1F and G). The analysis of the relationship between UHRF1 expression and clinicopathological features is shown 
Table II. Relationship between UHRF1 expression and clinicopathological findings.

\begin{tabular}{|c|c|c|c|}
\hline & $\begin{array}{l}\text { High expression }(\mathrm{n}=152) \\
\mathrm{n}(\%)\end{array}$ & $\begin{array}{l}\text { Low expression }(\mathrm{n}=79) \\
\mathrm{n}(\%)\end{array}$ & P-value \\
\hline \multicolumn{4}{|l|}{ Gender } \\
\hline Male & $84(61.8)$ & $52(38.2)$ & 0.12 \\
\hline Female & $68(71.6)$ & $27(28.4)$ & \\
\hline Median age (years) & 66.9 (range 33-89) & 64.3 (range $24-85$ ) & 0.12 \\
\hline Tumor location & & & 0.008 \\
\hline Right hemicolon & $61(77.2)$ & $18(22.8)$ & \\
\hline Left hemicolon & $91(59.9)$ & $61(40.1)$ & \\
\hline Tumor differentiation & & & 0.47 \\
\hline Differentiated & $134(66.6)$ & $67(33.4)$ & \\
\hline Undifferentiated & $18(60)$ & $12(40)$ & \\
\hline Depth of invasion & & & 0.051 \\
\hline $\mathrm{T} 1$ & $4(50)$ & $4(50)$ & \\
\hline $\mathrm{T} 2$ & $26(57.8)$ & $19(42.2)$ & \\
\hline $\mathrm{T} 3$ & $109(67.3)$ & $53(32.7)$ & \\
\hline $\mathrm{T} 4$ & $13(81.3)$ & $3(18.7)$ & \\
\hline Lymph node metastasis & & & 0.36 \\
\hline No & $88(63.8)$ & $50(36.2)$ & \\
\hline N1 & $41(70.7)$ & $17(29.3)$ & \\
\hline $\mathrm{N} 2$ & $12(57.1)$ & $9(42.9)$ & \\
\hline N3 & $7(100)$ & $0(0)$ & \\
\hline UICC stage & & & 0.45 \\
\hline 0 & $2(40)$ & $3(60)$ & \\
\hline I & $22(61.1)$ & $14(38.9)$ & \\
\hline II & $57(67.1)$ & $28(32.9)$ & \\
\hline III & $46(68.7)$ & $21(31.3)$ & \\
\hline IV & $25(65.8)$ & $13(34.2)$ & \\
\hline
\end{tabular}

Table III. Relationship between UHRF1 and E2F-1 expression in 231 colorectal cancer patients.

\section{E2F-1}

$\overline{\text { High } \quad \text { Low }}$

\begin{tabular}{lll}
\hline UHRF1 & & \\
High & 85 & 67 \\
Low & 14 & 65 \\
\hline
\end{tabular}

$\mathrm{P}<0.0001$

in Table II. UHRF1 expression was correlated with the anatomical location of the tumor. The positive cases of UHRF1 expression in right hemicolon cancer were significantly more frequent than those in left hemicolon cancer $(\mathrm{P}=0.008)$. Moreover, high UHRF1 expression showed an association with depth of invasion $(\mathrm{P}=0.051)$. Correlations between UHRF1 expression and other factors, including gender, age, lymphatic invasion, venous invasion, lymph node metastasis or stage, were not observed. The 5-year survival outcome did not differ between patients with a high UHRF1 expression compared to those with low UHRF1 expression (data not shown).

Correlation between UHRF1 and E2F-1 expression. High E2F-1 expression was detected immunohistochemically in 99 (42.9\%) colorectal cancer specimens (Fig. 1H and I). When the association between UHRF1 and E2F-1 expression was analyzed, a statistically significant difference was noted $(\mathrm{P}<0.0001)$. (Table III).

Knockdown of UHRF1 induces cellular growth inhibition. UHRF1 expression was examined by western blotting in 12 human colon cancer cell lines (Fig. 2A). Of these cell lines, the HCT116 and SW620 cells, which express UHRF1 at relatively high and low levels respectively, were used for knockdown experiments. First, the effect of UHRF1 siRNA was validated. The expression level of UHRF1 was significantly decreased by UHRF1 siRNA treatment compared with scrambled siRNA at both the protein and mRNA levels (Fig. 2B and C). A morphological change was not observed in all of the knocked-down UHRF1 cells. Significant suppression of cellular proliferation 
$\mathbf{A}$

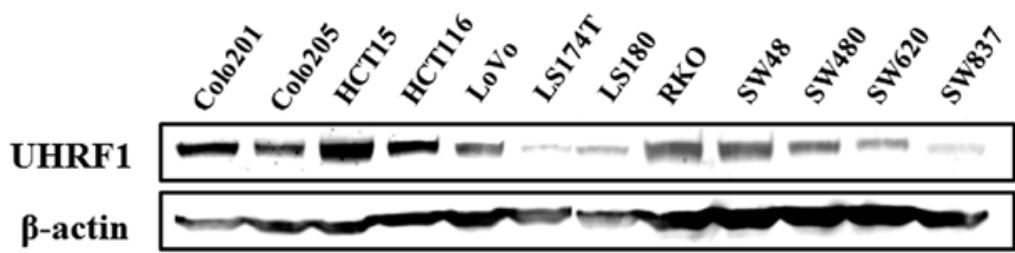

B

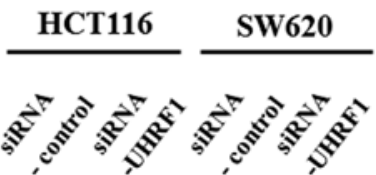

C

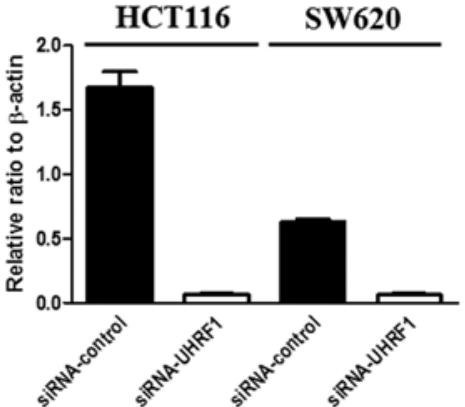

Figure 2. UHRF1 protein expression in 12 colon cancer cell lines and validation of the knockdown effect of the UHRF1 siRNA. (A) UHRF1 protein expression was determined by western blotting using total cell lysates prepared from 12 colon cancer cell lines. Thirty micrograms was applied to each gel. $\beta$-actin was used as the internal control. (B) HCT116 and SW620 cells were transfected with 40 nM UHRF1 siRNA (siRNA-UHRF1) or 40 nM control siRNA (siRNAcontrol), and incubated for $48 \mathrm{~h}$. UHRF1 expression was examined by western blotting. $\beta$-actin was probed as an internal control. (C) RT-PCR was performed for detection of UHRF1 mRNA using the same samples as above. UHRF1 expression levels were normalized by $\beta$-actin mRNA expression levels. Columns represent an average of 3 independent experiments; Bars, SD.

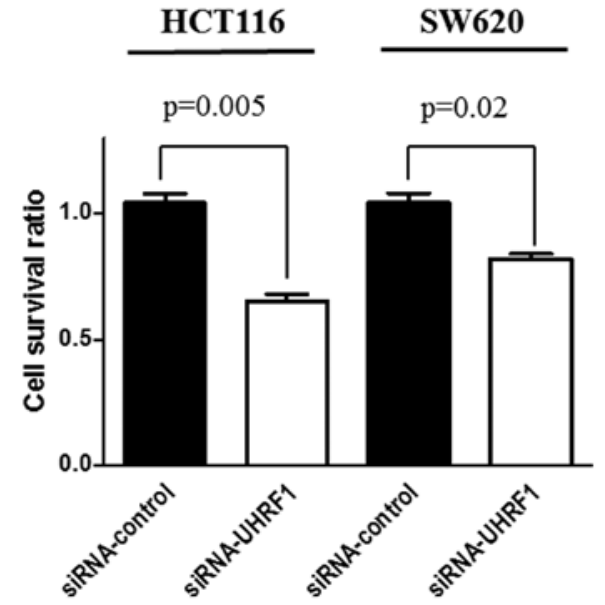

Figure 3. Effect of the UHRF1 siRNA on cell growth. HCT116 and SW620 cells were transfected with $40 \mathrm{nM}$ siRNA-UHRF1 or $40 \mathrm{nM}$ siRNA-control, and incubated for $48 \mathrm{~h}$. The analysis was performed using Cell Counting Kit-8. Statistical analysis was performed using the Student's t-test. Columns represent an average of 3 independent experiments; Bars, SD.

was observed in both HCT116 and SW620 cells treated with UHRF1 siRNA compared with scrambled siRNA $(\mathrm{P}=0.005$, $\mathrm{P}=0.02$, respectively) (Fig. 3).

\section{Discussion}

Previous studies have shown that UHRF1 expression is constantly upregulated in proliferating cultured cancer cells and various types of cancer (5-12). Therefore, enhanced UHRF1 expression in cancer cells is considered to be associated with cellular proliferation. To the best of our knowledge, we demonstrated for the first time that UHRF1 expression was upregulated in approximately two-thirds of all colon cancer cases. Moreover, increased UHRF1 expression was observed in a number of specimens of adenoma, but not in colonic normal mucosa. Our findings indicate that UHRF1 expression is upregulated at an early stage of colorectal carcinogenesis and may be involved in cellular proliferation.

Regarding clinical significance of enhanced UHRF1 expression in colorectal cancer, the frequency of high UHRF1 expression in cancer tissue in the right hemicolon was higher than that in the left hemicolon. It has been implicated that cancer of the right (RCC) and left colon (LCC) has a differing prevalence at varying ages, in high- and low-incidence regions, as well as in males and in females. There is also a difference in clinical presentation, prognosis and possibly in genetic and environmental epidemiology $(25,26)$. For examples, RCC is more common in females, LCC in males (25), response to 5 -fluorouracil treatment is significantly better in RCC (27), nuclear $\beta$-catenin and p53 are expressed to a greater extent in rectal compared with proximal cancer (28), expression of cytoplasmic c-erbB2, epidermal growth factor receptor (EGFR), proliferating cell nuclear antigen (PCNA), and dipeptidylpeptidase IV (DPP IV) is higher in RCC compared with LCC (28). Distal tumors display a higher frequency of $17 \mathrm{p}$ and $18 \mathrm{q}$ allelic loss, p53 accumulation, c-myc expression and aneuploidy compared to proximal tumors (29). Since the expression of UHRF1 was especially high in right hemicolon cancer, UHRF1 may be one of the factors that determine these features of RCC. Furthermore, upregulated UHRF1 tended to be involved with deeper invasion, suggesting that UHRF1 may be involved in malignant transformation of colon cancer. Statistical correlations were not observed for gender, age, lymph duct invasion, vessel invasion, lymph node metastasis and stage in colorectal cancer. Survival outcome for each stage had no significance between UHRF1positive and -negative cases. These results suggest that UHRF1 expression did not directly affect the metastasis and prognosis 
of colon cancer, but UHRF1 may increase the possiblity of DNA damages or replication errors, which cause the malignant transformation, by promoting speed of cell cycles.

It has been reported that E2F-1 upregulates UHRF1 transcription through binding to E2F-1 binding consensus sequences found on the UHRF1 promoter $(6,7,24)$. We then investigated the relationship between the expression levels of UHRF1 and E2F-1 in clinical cases. Our results showed that the number of patients with high UHRF1 and high E2F-1 expression is larger than that of others. Therefore, UHRF1 expression was significantly associated with E2F-1 expression in colorectal cancer.

UHRF1 plays an essential role in G1/S transition, and knockdown of UHRF1 suppresses cellular proliferation $(7,14)$. We also demonstrated that knockdown of UHRF1 inhibited cellular proliferation in colon cancer cell lines. These facts suggest that UHRF1 may be a therapeutic target for colorectal cancer patients with high UHRF1 expression. Knockdown of E2F-1 has also been reported to decrease UHRF1 expression (14). Considering clinical cases, UHRF1 can be considered a better therapeutic target of colon cancer than E2F-1 because the rate of UHRF1-positive cases in colon cancer was higher compared to E2F-1 (65.8 and 42.9\%, respectively). Furthermore, UHRF1 interacting proteins such as DNMTs and HDACs are satisfactory targets of anticancer drugs (13) and a combination therapy of an UHRF1 inhibitor with these drugs may be more effective.

In conclusion, we found that UHRF1 expression was upregulated and involved in the cellular proliferation of colorectal cancer. Particularly, UHRF1 expression appears to be associated with carcinogenesis of the right hemicolon. Based on our novel findings, UHRF1 is a therapeutic target for colorectal cancer.

\section{References}

1. Saltz LB, Clarke S, Díaz-Rubio E, Scheithauer W, Figer A, Wong R, Koski S, Lichinitser M, Yang TS, Rivera F, et al: Bevacizumab in combination with oxaliplatin-based chemotherapy as first-line therapy in metastatic colorectal cancer: a randomized phase III study. J Clin Oncol 26: 2013-2019, 2008.

2. Bokemeyer C, Bondarenko I, Makhson A, Hartmann JT, Aparicio J, de Braud F, Donea S, Ludwig H, Schuch G, Stroh C, et al: Fluorouracil, leucovorin, and oxaliplatin with and without cetuximab in the first-line treatment of metastatic colorectal cancer. J Clin Oncol 27: 663-671, 2009.

3. Douillard JY, Siena S, Cassidy J, Tabernero J, Burkes R, Barugel M, Humblet Y, Bodoky G, Cunningham D, Jassem J, et al: Randomized, phase III trial of panitumumab with infusional fluorouracil, leucovorin, and oxaliplatin (FOLFOX4) versus FOLFOX4 alone as first-line treatment in patients with previously untreated metastatic colorectal cancer: the PRIME study. J Clin Oncol 28: 4697-4705, 2010.

4. Karapetis CS, Khambata-Ford S, Jonker DJ, O'Callaghan CJ, Tu D, Tebbutt NC, Simes RJ, Chalchal H, Shapiro JD, Robitaille S et al: K-ras mutations and benefit from cetuximab in advanced colorectal cancer. N Engl J Med 359: 1757-1765, 2008

5. Hopfner R, Mousli M, Jeltsch JM, Voulgaris A, Lutz Y, Marin C, Bellocq JP, Oudet P and Bronner C: ICBP90, a novel human CCAAT bonding protein, involved in the regulation of topoisomerase II alpha expression. Cancer Res 60: 121-128, 2000.

6. Mousli M, Hopfner R, Abbady AQ, Monté D, Jeanblanc M, Oudet P, Louis B and Bronner C: ICBP90 belongs to a new family of proteins with an expression that is deregulated in cancer cells. Br J Cancer 89: 120-127, 2003.

7. Unoki M, Nishidate T and Nakamura Y: ICBP90, an E2F-1 target, recruits HDAC1 and binds to methyl-CpG through its SRA domain. Oncogene 23: 7601-7610, 2004.

8. Unoki M, Daigo Y, Koinuma J, Tsuchiya E, Hamamoto R and Nakamura Y: UHRF1 is a novel diagnostic marker of lung cancer. Br J Cancer 103: 217-222, 2010.
9. Crnogorac-Jurcevic T, Gangeswaran R, Bhakta V, Capurso G, Lattimore S, Akada M, Sunamura M, Prime W, Campbell F, Brentnall TA, et al: Proteomic analysis of chronic pancreatitis and pancreatic adenocarcinoma. Gastroenterology 129: 1454-1463, 2005.

10. Oba-Shinjo SM, Bengtson MH, Winnischofer SM, Colin C, Vedoy CG, de Mendonça Z, Marie SK and Sogayar MC: Identification of novel differentially expressed genes in human astrocytomas by cDNA representational difference analysis. Brain Res Mol Brain Res 140: 25-33, 2005.

11. Lorenzato M, Caudroy S, Bronner C, Evrard G, Simon M, Durlach A, Birembaut P and Clavel C: Cell cycle and/or proliferation markers: what is the best method to discriminate cervical high-grade lesions? Hum Pathol 36: 1101-1107, 2005.

12. Unoki M, Kelly JD, Neal DE, Ponder BA, Nakamura Y and Hamamoto R: UHRF1 is a novel molecular marker for diagnosis and the prognosis of bladder cancer. Br J Cancer 101: 98-105, 2009.

13. Unoki M, Brunet J and Mousli M: Drug discovery targeting epigenetic codes: the great potential of UHRF1, which links DNA methylation and histone modifications, as a drug target in cancers and toxoplasmosis. Biochem Pharmacol 78: 1279-1288, 2009.

14. Arima Y, Hirota T, Bronner C, Mousli M, Fujiwara T, Niwa S, Ishikawa $\mathrm{H}$ and Saya $\mathrm{H}$ : Down-regulation of nuclear protein ICBP90 by p53/p21Cip1/WAF1-dependent DNA-damage checkpoint signals contributes to cell cycle arrest at G1/S transition. Genes Cells 9: 131-142, 2004.

15. Trotzier MA, Bronner C, Bathami K, Mathieu E, Abbady AQ, Jeanblanc M, Muller CD, Rochette-Egly C and Mousli M: Phosphorylation of ICBP90 by protein kinase A enhances topoisomerase II alpha expression. Biochem Biophys Res Commun 319: 590-595, 2004.

16. Bostick M, Kim JK, Estève PO, Clark A, Pradhan S and Jacobsen SE: UHRF1 plays a role in maintaining DNA methylation in mammalian cells. Science 317: 1760-1764, 2007.

17. Arita K, Ariyoshi M, Tochio H, Nakamura Y and Shirakawa M: Recognition of hemi-methylated DNA by the SRA protein UHRF1 by a base-flipping mechanism. Nature 455: 818-821, 2008.

18. Avvakumov GV, Walker JR, Xue S, Li Y, Duan S, Bronner C, Arrowsmith $\mathrm{CH}$ and Dhe-Paganon S: Structural basis for recognition of hemi-methylated DNA by the SRA domain of human UHRF1. Nature 455: 822-825, 2008.

19. Hashimoto H, Horton JR, Zhang X, Bostick M, Jacobsen SE and Cheng X: The SRA domain of UHRF1 flips 5-methylcytosine out of the DNA helix. Nature 455: 826-829, 2008.

20. Sharif J, Muto M, Takebayashi S, Suetake I, Iwamatsu A, Endo TA, Shinga J, Mizutani-Koseki Y, Toyoda T, Okamura K, et al: The SRA protein Np95 mediates epigenetic inheritance by recruiting Dnmt1 to methylated DNA. Nature 450: 908-912, 2007.

21. Kim JK, Estève PO, Jacobsen SE and Pradhan S: UHRF1 binds G9a and participates in p21 transcriptional regulation in mammalian cells. Nucleic Acids Res 37: 493-505, 2009.

22. Jin W, Chen L, Chen Y, Xu SG, Di GH, Yin WJ, Wu J and Shao ZM: UHRF1 is associated with epigenetic silencing of BRCA1 in sporadic breast cancer. Breast Cancer Res Treat 123: 359-373, 2010.

23. Meilinger D, Fellinger K, Bultmann S, Rothbauer U, Bonapace IM, Klinkert WE, Spada F and Leonhardt H: Np95 interacts with de novo DNA methyltransferases, Dnmt3a and Dnmt3b, and mediates epigenetic silencing of the viral CMV promoter in embryonic stem cells. EMBO Rep 10: 1259-1264, 2009.

24. Abbady AQ, Bronner C, Bathami K, Muller CD, Jeanblanc M, Mathieu E, Klein JP, Candolfi E and Mousli M: TCR pathway involves ICBP90 gene down-regulation via E2F binding sites. Biochem Pharmacol 70: 570-579, 2005.

25. Distler P and Holt PR: Are right- and left-sided colon neoplasms distinct tumors? Dig Dis 15: 302-311, 1997.

26. Birkenkamp-Demtroder K, Olesen SH, Sørensen FB, et al: Differential gene expression in colon cancer of the caecum versus the sigmoid and rectosigmoid. Gut 54: 374-384, 2005.

27. Elsaleh H, Joseph D, Grieu F, Zeps N, Spry N and Iacopetta B: Association of tumour site and sex with survival benefit from adjuvant chemotherapy in colorectal cancer. Lancet 355: $1745-1750,2000$

28. Kapiteijn E, Liefers GJ, Los LC, et al: Mechanisms of oncogenesis in colon versus rectal cancer. J Pathol 195: 171-178, 2001

29. Fric P, Sovová V, Sloncová E, Lojda Z, Jirásek A and Cermák J: Different expression of some molecular markers in sporadic cancer of the left and right colon. Eur J Cancer Prev 9: 265-268, 2000 . 